die Inzision des Parenchyms zielbewußt gegen nephritische Blutungen und Schmerzen ins Feld geführt.

Harrison hatte im Jahre 1896 die Nierenspaltung in bestimmten Fällen zur Beseitigung der Albuminurie ausgeführt; aber auch er beschränkte sich auf bestimmte Indikationen. Er wollte in Analogie mit der Iridektomie bei Glaukom durch die Inzision der Niere eine Druckentlastung des Organs erreichen; er operierte nur bei steigendem Eiweifgehalt während einer akuten Nephritis, bei starker Oligurie und bei Komplikationen durch Herzaffektionen. Ein Urteil über den Wert seiner Methode, welche namentlich in Pousson einen warmen Fürsprecher gefunden hat, deren Indikation hinwiederum Pel (Amsterdam) möglichst einengen möchte, läßt sich zurzeit noch nicht abgeben.

Edebohls ging einen groben Schritt weiter, indem er ganz unabhängig von dem Vorhandensein oder Fehlen quälender oder gefahrdrohender Symptome jeden Fall von Brightscher Krankheit mittels Dekapsulation zu operieren riet, sofern er nicht unheilbare Komplikationen aufwies und seine Lebensdauer ohne Operation mutmaßlich nicht weniger als ein Monat betragen würde.

Edebohls hat zwei Serien seiner operierten Fälle veröffentlicht. In seiner ersten Publikation, welche 19 Fälle umfaßt, bezeichnet er neun Fälle als vollständig geheilt, nachdem er die Kranken ein bis acht Jahre nach der Operation dauernd kontrolliert hatte; im vorigen Jahre konnte er bereits über 51 Operierte berichten, mit folgenden Resultaten:

14 Todesfälle $=27 \%$ (davon sieben innerhalb der ersten 17 Tage, die andere Hälfte nach zwei Monaten bis acht Jahren); 22 Besserungen $=43 \%$; ein Rezidiv nach vierjähriger Heilung; zwei unbeeinflußt; drei verschollen; neun dauernd geheilt $=18 \%$

Dabei verlangt Edebohls von einem geheilten Falle, daß er

Aus der chirurgischen Abteilung des Krankenhauses der Jüdischen Gemeinde in Berlin. (Direktor: Prof. Dr. J. Israel.)

\section{Klinische Erfahrungen über die Dekapsulation der Nieren beim Morbus Brightii.')}

\section{Von Dr. Paul Rosenstein, I. Assistenzarzt.}

Als Edebohls im Jahre 1901 mit seinen Angaben über staunenswerte Heilerfolge bei Morbus Brightii durch die Dekapsulation der Nieren an die Oeffentlichkeit trat, erregte er die Aufmerksamkeit aller interessierten Kreise. Sowohl innere Aerzte wie Chirurgen konnten sich der Pflicht nicht entziehen, diese neue Art der Behandlung eines bisher unbeeinflufbaren Leidens trotz einer berechtigten Skepsis zu prüfen.

Edebohls hatte eine Anzahl von Frauen, welche mit Wanderniere behaftet waren, der Nephropexie unterzogen und dabei beobachtet, daß die die Wanderniere begleitende chronische Nephritis nach der Beseitigung der abnormen Mobilität ausheilte. Diese Erfahrung war anderen Chirurgen schon lange bekannt, neu waren nur die daraus von Edebohls gezogenen Schlüsse. Er bezog das Verschwinden des entzündlichen Prozesses weniger auf die Fixation der Nieren als auf die mit seiner Methode der Befestigung verbundene partielle Abziehung der fibrösen Kapsel und kam, veranlaßt durch diese unabsichtlich erzielte̊ Nebenfrucht seiner Operation, auf die Idee, die. Enthülsung der Nieren als Heilmittel des Morbus Brightii zu versuchen, auch wenn keine abnorme Mobilität vorhanden war.

Das war ein neuer Schritt auf dem Gebiete der Nierenchirurgie; wohl hatten schon früher Chirurgen mit bestem $\mathrm{Er}$ folge den Feldzug gegen gewisse, . mit der chronischen Nephritis verbundene Krankheitsäuferungen eröffnet, ein Vorgehen, welches sich hauptsächlich an den Namen Israel knüpft. Niemals aber waren diese Eingriffe, welche wesentlich in der Inzision des Nierenparenchyms bestanden, mit der Absicht unternommen worden, die Nephritis zu heilen, sondern stets war das Ziel der Operation die Beseitigung von Koliken, Schmerzen, Blutungen oder Anurie, also ungewöhnlichen, peinigenden oder gefahrdrohenden Begleitzuständen mancher Arten von Nierenentzündung. Die Heilwirkung der Inzision bei nephritischen Blutungen und Schmerzen war zufällig entdeckt worden; man hatte auf Grund der Symptome Stein, Tuberkulose oder Neubildung erwartet, die Niere probatorisch inzidiert, aber nicht gefunden, was man vermutet hatte; dennoch hatte der Eingriff häufig die Blutungen und die Schmerzen beseitigt. Als Resultat genauerer Untersuchungen stellte sich heraus, daß als anatomische Grundlage der Blutungen und Schmerzen häufig eine chronische Enzündung der Nieren gefunden wurde. Erst nach dieser Erkenntnis wurde

2) Auszugsweise vorgetragen am dritten Sitzungstage des XXX11l. Kongresses der Deutschen Gesellsctraft für Chirurgie zu Berlin (8. April 1904). mindestens sechs Monate hindurch einen von Eiweiß und Nierenelementen freien Urin aufweist, die genügende tägliche Harnmenge liefert und keinerlei sonstige Symptome der Brightschen Krankheit zeigt.

Wenn Edebohls bei einer so strengen Abgrenzung des Heilungsbegriffes über $18 \%$ Dauerheilungen und $43 \%$ Besserungen berichten konnte, so mußte diese günstige Statistik auffordern, an einzelnen Fällen, bei denen jede andere Therapie versagt hatte, einen therapeutischen Versuch in der vorgeschlagenen Richtung zu machen; dieser Erwägung zufolge hat mein Chef, Herr Prof. Israel, die Dekapsulation beider Nieren seither in sechs Fällen von sicherem Morbus Brightii ausgeführt.

Unsere Resultate entsprechen den Edebohlsschen leider nicht: ein Patient ist durch die Operation gestorben; einer befindet sich zur Zeit erheblich schlechter; zwei sind unbeeinflußt; zwei sind in gewisser Hinsicht gebessert; 0 ist geheilt.

Der Fall, welcher durch die Operation gestorben ist, betraf ein junges Mädchen von 19 Jahren, welches seit drei Jahren an Morbus Brightii litt; sie wurde in zwei Sitzungen operiert, erst die rechte, dann nach drei Monaten die linke Niere dekapsuliert; jeder Operation folgte ein urämischer Anfall; der erste ging nach zwei Tagen zurück; der zweite führte aber acht Tage nach der zweiten Operation zum Tode im Koma. Es ist sicher, daß hier die Operation als solche den Tod verursacht hat, denn die urämischen Anfälle folgten prompt der Dekapsulation jeder Seite, während vorher bei der Kranken niemals urämische Symptome bestanden hatten, obwohl vor der ersten Operation eine Erniedrigung des Blutgefrierpunktes von 0,62 sich ergab. Noch ein zweiter Fall (No. 6), welcher durch Hydronephrose einer Seite kompliziert war, wurde in zwei vier Wochen voneinander gelegenen Zeitabständen operiert, die übrigen vier Patienten wurden in einer Sitzung beiderseitig, respektive an beiden Nieren (cf. Fall 5) dekapsuliert.

Ich lasse die sechs Krankengeschichten in möglichst gedrängter Kürze folgen:

Krankengeschichten: Fall 1. Fräulein R., 19 Jahre alt, aufgenommen am 1. Juli 1902, gestorben am 19. Dezember 1902

Anamnese: Patientin erkrankte aus vollem Wohlbefinden vor drei Jahren unter Uebelkeit, Kopfschmerzen und Erbrechen; dabei schwollen gleichzeitig Hände, Füße und Gesicht an. Diese Beschwerden wechselten bis jetzt in ihrer Intensität.

Status praesens: Auffallende Blässe der Schleimhäute. Keine Oedeme. Gedunsenheit des Gesichts. Hypertrophie des linken Ven. trikels. Puls klein, mäßig gespannt. $215 \mathrm{ccm}$ Blutdruck. Blutgefrierpunkt $=0,62$. Urin sauer, Albumen $51 / 2 \%$; im Sediment granulierte und hyaline Zylinder, rote und weiße Blutkörperchen. 24 stündige Menge 1300, spezifisches Gewicht 1010. Erste Operation in Aethernarkose 9. Juli 1902 (Dekapsulation der rechten Niere). Fibröse Kapsel leicht abziebar; Niere etwas vergrößert, Oberfläche glatt, außer einzelnen gelben Flecken nichts Abnormes.

11. Juli. Patientin hat mehrere urämische Krampfanfälle gehabt; 
ist besinnungslos; starkes Erbrechen, sehr frequenter $(150-160)$, gespannter Puls, Temperatur $39,2^{\circ}$. Urinmenge seit gestern $500 \mathrm{ccm}$.

13. Juli. Heute kehrt das Bewußtsein wieder; seit gestern keine Anfälle mehr. Nachlaß aller urämischen Symptome.

5. August. Patientin hat inzwischen noch. verschiedene Male kleine urämische Attacken gehabt (Bewußtseinsstörung, Erbrechen, geringe Urinmenge). Heute hat sie sich so weit erholt, daß sie das Bett verlassen kann.

21. August. Albumengehalt sehr wechselnd, zwischen 4 und $9 \%$ bei einer durchschnittlichen Menge von $\mathrm{zw}^{\mathrm{e}} \mathrm{ei}$ bis drei Litern. Im Sediment viel Zylinder und rote Blutkörperchen. Allgemeinbefinden subjektiv gut.

8. Oktober. Zweite Operation (Narkose mit Billrothscher Mischung). Dekapsulation der linken Niere. Die Niere ist bedeutend härter als rechts, einzelne Stellen eingezogen und mit der fibrösen Kapsel verwachsen. Unterer Nierenpol besonders blaß und hart. Bis zum 13. Oktober gutes Befinden. Dann zum ersten Male Erbrechen und auffallend blasse Gesichtsfarbe.

15. Oktober. Patientin wird bew'ußtlos; andanerndes Erbrechen. 16. Oktober. Heute vollständiges Koma mit Erbrechen und Krampfanfällen. Urinmenge stark vermindert.

19. Oktober. Unter zunehmender Schwäche Exitus letalis im Koma.

Fall 2. Herr H., 29 Jahre alt, aufgenommen am 29. Januar 1903, entlassen 29. März 1903.

Anamnese: Beide Eltern leiden an Diabetes. Vor 15 Jahren hatte Patient Typhus. Oktober 1902 stellte sich bei vollem Wohlbefinden Oedem der Knöchel ein; ein hinzugezogener Arzt konstatierte Nephritis mit 10\% Albumen. Schnelle Zunahme der Oedeme trotz Bettruhe. In letzter Zeit Diarrhöen und starke, profuse Schweiße.

Status praesens: Sehr blasser junger Mann. Oedem der Hände und Füße; Gedunsenheit des Gesichts. Geringe Hypertrophie des linken Ventrikels. $\delta=0,76$.

Urinmenge 1200; spezifisches Gewicht 1018. Albumen $12 \%$. Sediment enthält viel granulierte Zylinder, Epithelien, weiße und wenig rote Blutkörperchen. Reaktion sauer.

2. Februar 1903. Operation in kombinierter Chloroform-Aethernarkose. Dekapsulation beider Nieren. Starkes penirenales (lımbales) Oedem beiderseits. Rechte Niere klein, weich, etwas zyanotisch, linke Niere sehr vergrößert, blaurot, enorm gespannt. Fibröse Kapsel beiderseits sehr leicht abziehbar.

29. März 1902. Entlassung. Oedeme wie vorher, Albumen $10 \%$, Sediment unverändert. 24 stündige Urinmenge 1300 , spezifisches Gewicht 1018. Letzte Nachricht 15 Monate nach der Operation: Oedeme des ganzen Körpers, welche zur dauernden Bettruhe $\mathrm{zwingen.}$

Fall. 3. Frau W., 27 Jahre alt; aufgenommen 25. November 1903, entlassen 29. Dezember 1903.

A namnese: Abort vor fünf und vor drei Jahren; damals als Ursache Nierenentzündung festgestellt. Seit vier Jahren Gelenkschmerzen (Knie, Hand, Fuß); außerordentlich leichtes Auftreten von Oedemen, namentlich bei Abkühlung. Vor drei Wochen zur Kur nach Karlsbad geschickt; daselbst Inunktionskur mit grauer Salbe. Wegen starker Verschlechterung des Befindens und Quecksilbervergiftung Aufnahme ins Krankenhaus. Viel Kopfschmerzen.

Status praesens: Keine Oedeme. Stomatitis mercurialis mit Ulzerationen der ganzen Mundhöhle; gangränöse Verfärbung der Zunge. Foetor ex ore. Arhythmia cordis. Hypertrophie des linken Ventrikels. Urinmenge 1600, spezifisches Gewicht 1010 sauer. Im Sediment zahlreiche hyaline und granulierte Zylinder, Nierenepithelien und Leuko zyten. Albumen $1 \frac{1}{2} \%$ \%

Beiderseits chorioretinale atrophische Herde von großer Ausdehnung. (Dr. Feilchenfeld.) Nach Ausheilung der Stomatitis 2. November Operation in Aethernarkose. Dekapsulation beider Nieren. Rechte Capsula propria trübe, schwer abziehbar, an einzelnen zirrhotischen Stellen der Niere fest verwachsen. Linke Niere aplastisch, so groß wie die Niere eines 5 jährigen Kindes. Kapsel verdickt und trüber als rechts, inniger verwachsen; ausgesprochene Granularatrophie.

29. Dezember. Entlassung. Nachlaß der Kopfschmerzen. Urinbefund wie vorher.

Letzte Nachricht Mai 1904: Viel rheumatische Beschwerden; sonst unverändert.

Fall 4. Frl. Sch., 28 Jahr alt. Aufgenommen 10. November 1903. Entlassen 11. Dezember 1903.

Anamnese: Vor 15 Jahren Scharlach und akute Nierenentzündung, welche vorüberging. Vor zehn Jahren akuter Gelenkrheumatismus. Vor zwei Jahren ohne Ursache Rückenschmerzen, Oedeme der Beine und häufiger Urindrang bei sehr geringer Menge. Im Dezember 1902, Februar und Mai 1903 urämische Anfälle mit Oligurie, Erbrechen und Bewußtlosigkeit. Zur Zeit viel Kopfschmerzen, Flimmern vor den Augen, sonst subjektives Wohlbefinden.

Status praesens: Keine Oedeme. Etwas pastöses Aussehen. Hypertrophia cordis. Linke Niere stark vergrößert, hart, schmerzhaft bei Berührung. Rechte Niere palpabel, nicht vergrößert, ohne Druckempfindlichkeit. Myopischer Astigmatismus. $\delta=0,55$.

Urin sauer, 24stündige Menge 1300; spezifisches Gewicht 1015.
Albumen $2-3 \%$. Im Sediment viel granulierte Zylinder, weniger Eiterkörperchen.

16. November. Operation in Aethernarkose. Dekapsulation beider Nieren. Rechte Niere kleiner als gewöhnlich, fötal gelappt. Capsula propria derb, trübe, adhärent mit der Fettkapsel, aber von der Niere leicht abziehbar. Oberfläche der Niere blutend; gelbgesprenkelte, feinfleckige Oberfläche (Verfettungen). Linke Niere ist vergrößert, sonst genau wie rechts.

Letzte Untersuchung Mai 1904. Keine Kopfschmerzen, kein Flimmern vor den Augen; subjektives Wohlbefinden. Urinbefund unverändert; Albumen 5\%; 24stiindige Menge 1800; spezifisches Gewicht 1015. Im Sediment viel granulierte Zylinder, Epithelien und weiße Blutkörperchen.

Fall 5. Herr Ch., 17 Jahre alt. Aufgenommen am 28. September 1903. Entlassen 9. Februar 1904.

Anamnese: Vor neun Monaten acht Tage dauernde Hämaturie; fünf Tage darauf Anschwellen der linken Gesichtshälfte (Parotisabszeß). Inzision im April 1903. Hohe Temperaturen (39 $)$ bis zu einer zweiten Inzision, welche reichlich Eiter entleerte. Urin seither dauernd trübe. Status praesens: Keine Oedeme, aber starke Gedunsenheit und Blässe des Gesichts. Eiternde Parotisfistel. Hypertrophia cordis. Nieren nicht palpabel.

Urin sauer, 24stündige Menge 750, spezifisches Gewicht 1021. Albumen $1 \frac{1}{2} \%$. Im Sediment viel hyaline und granulierte Zylinder, weiße und sehr viel rote Blutkörperchen, Blutschatten.

30. September: Exkochleation der Parotisfistel in Chloroformnarkose; darauf drei Tage anhaltende, heftige Hämaturie.

14. Dezember: Operation in Skopolamin-Morphilumnarkose; etwas Chloroforminhalation zur vollständigen Narkose nötig. Dekapsulation. Rechts ist die Nierennische leer; es wird rechts keine Niere gefunden. Links liegen beide Nieren, mit ihren Polen direkt ineinander übergehend, nur durch eine Einschnürung voneinander erkennbar abgesetzt. Die Niere ist bläulich verfärbt, sehr schlaff; leicht abziebare Kapsel. Letzte Nachricht April 1904: Zustand gänzlich unbeeinflußt.

Fall 6. Herr W., 35 Jahre alt. Aufgenommen 11. Oktober 1903. Entlassen 11. Dezember 1903.

Anamnese: Mutter vor zwei Jahren an Morbus Brightii gestorben, ebenso Großvater. Vor 20 Jahren chronische Meningitis. Vor 19 Jahren Kantharidinvergiftıng und Hämaturie. Seither Urin trïbe und schleimig. Von Zeit zu Zeit Fieber und Eiterentleerung mit dem Urin. Mehrfach suburämische Zustände mit Kopfschmerzen nnd Bewußtseinsstörung. Häufig Uebelkeiten, Krämpfe, Lendenschmerzen.

Status praesens: Keine Oedeme. Hypertrophia cordis.

Urinmenge 1700, spezifisches Gewicht 1011; Albumen 1\%. Im Sediment Leukozyten und mit Epithelien besetzte Zylinder; einige Blutschatten, Tripelphosphatkristalle.

17. Oktober: Patient bekommt plötzlich beim Essen krampfartige Zustände mit Opistotonus und vorübergehenden Lähmungen des rechten Armes und Beines; nach Wiederkehr des Bewußtseins starke Rückenschmerzen und kolikartige Schmerzen der rechten Nierengegend.

26. Oktober: Operation in Aethernarkose. Dekapsulation der rechten Niere; diese ist auf $2 / 3$ des normalen Volumens verkleinert, groblappig, zirrhotisch. Capsula fibrosa trübe, größtenteils verwachsen mit der Nierensubstanz, welche stark blutet.

15. November: Patient steht in der dritten Woche nach der Operation auf, erholt sich aber sehr langsam; ist wieder gut bei Kräften.

23. November: Patient klagt zuletzt über viel Schmerzen in der linken Niere und vorübergehende Uebelkeiten.

Zweite Operation: Dekapsulation der linken Niere; dieselbe ist sehr geschrumpft, schlaff; an der Oberfläche ein pflaumengroßer, fluktuierender Buckel. Hydronephrotisch erweitertes Becken.

11. Dezember: Entlassung mit $1 / 4-1 / 2 \%$ Albumen.

Letzte Nachricht April 1904, daß das Leiden fortschreitet (Kopfschmerzen, Oedeme)

Wie sich aus den Krankenges chichten ergibt, beträgt unsere Beobachtungsdauer 5 bis 15 Monate nach der Operation; daher läpt sie schon ein gewisses Urteil zll. Bei einem Patienten hat das Leiden unbeeinflußt von dem Eingriff Fortschritte gemacht, so daf der Kranke sich zur Zeit in trostlosem Zustande befindet; er ist, wie Arzt und Angehörige berichten, durch Oedeme des ganzen Körpers dauernd ans Bett gefesselt. $\mathrm{Zwei}$ sind gänzlich unbeeinflußt geblieben, zwei Kranken sind in gewisser Hinsicht gebessert worden: während vorher bei einem von ihnen leicht Oedeme aufgetreten sein sollen, sind diese seither ausgeblieben, ferner schwanden in dem andern Falle die Kopfschmerzen und Flimmern vor den Augen, über welches vorher geklagt worden war; der lokale ProzeB blieb unverändert.

Wie erklärt sich nun der Unterschied der Edebohlsschen und unserer Ergebnisse? Die Antwort auf diese Frage ergibt sich aus einer sorgfältigen Kritik der Edebohlsschen Veröffentlichungen. Die von Edebohls durch die Dekapsulation geheilten Kranken haben sämtlich nicht an echter 
Brightscher Krankheit gelitten, sondern an entzündlichen Prozessen, die durch abnorme Mobilität der Nieren verursacht waren. Denn wenn wir die beiden Veröffentlichungen miteinander vergleichen, so verzeichnen wir die auffallende Tatsache, daß unter den ersten 19 Fällen mit 16 Wandernieren 9 geheilt worden sind, daß aber unter den nächsten 32 Kranken, unter denen nur 4 Wandernieren vorhanden waren, während die übrigen zweifellos Brightsche Krankheit darstellten, keine einzige neue Dauerheilung hinzukommt. Das heißt mit anderen Worten: alle Heilungen betreffen Wandernieren, keine einzige Heilung bei Morbus Brightii ohne abnorme Mobilität der Nieren. Und ferner: unter den ersten 19 Fällen sollen sich 9 von „einseitigem“ Morbus Brightii befunden haben, unter den 32 weiteren findet sich keine einseitige Erkrankung mehr. Die einseitigen Entzïndungen waren eben keine Brightsche Erkrankung, sondern Nephritis durch Wanderniere, denn einseitigen Morbus Brightii gibt es nicht; die Brightsche Krankheit ist eine toxische, durch gelöste Gifte erzeugte Nierenentzündung und ist daher stets doppelseitig. Die Dauerheilungen Edebohls' können wir also vollkommen ausschalten. Es bleiben noch weitere 11 Fälle, welche Edebohls als geheilt betrachtet, die aber nach seiner-eigenen Angabe wegen der zu kurzen Beobachtungsdauer noch nicht sicher sind; wir wollen sie zweckmäßig ganz aus der Diskussion lassen.

Wie steht es nun aber mit den 22 Besserungen?

Hier sind wir vollkommen auf Treu und Glauben angewiesen, denn keine Krankengeschichte wird uns als objektive Unterlage unseres Urteils unterbreitet. Edebohls veröffentlicht drei Berichte über die in den betreffenden Fällen erzielten Besser.ungen und fügt hinzu, daß die Berichte der übrigen als „gebessert“ Bezeichneten ungefähr den drei mitgeteilten entsprächen: Danach können wir die erreichten Besserungen nicht sehr hoch veranschlagen, denn in den Briefen heilit es z. B.: „Patient leidet viel an Kopfschmerzen und Verdauungsstörungen, obgleich die Beschaffenheit des Urins in beständiger Besserung begriffen ist", oder: „Die allgemeine Wassersucht war gänzlich verschwunden, aber in den letzten zwei Monaten sammelte sich wieder etwas Flüssigkeit in Abdomen an, und ich leide viel an Verdauungsstörungen“, und endlich der dritte, welcher berichtet, „dafl die Besserung durch akute Ausbrüche zuweilen gestört werde und er weit davon entfernt sei, sich wohl zu befinden". Und das sind sicherlich die Besten der „Gebesserten“, denn man darf annehmen, dall Edebohls zur Darlegung des gebesserten Zustandes gerade die günstigsten Berichte herausgegriffen hat. Danach nimmt sich die „günstige“ 'Statistik Edebohls' bei richtiger Beleuchtung ganz anders aus!

Schalten wir unter den 51 Kranken die 16 Wandernieren aus, die für die operative Heilbarkeit des Morbus Brightii nicht in Frage kommen, so bleiben 35; von diesen will Edebohls 11 wegen zu kurzer Beobachtungsdauer noch nicht verwerten, ferner fällt einer aus, welcher verschollen ist. Es bleiben also zur $\mathrm{Be}$ urteilung 23 Fälle von sicherem Morbus Brightii.

Davon sind: 6 operativ gestorben $=26 \%$, 0 geheilt, 8 ungebessert (und zwar sind von diesen 85 ungeheilt später an Nephritis gestorben; die übrigen 3 sind noch am Leben, aber krank), 9 sind "gebessert".

Wie weit wir diese neun nach unseren - Begriffen als gebessert bezeichnen würden, bleibt dahingestellt, denn Schwankungen im subjektiven wie objektiven Befinden sind bei Nephritikern etwas ganz Gewöhnliches. In der Tat besteht also zwischen der kritisch beleuchteten Edebohlsschen und unserer eigenen Statistik kein großer Unterschied; beide sind außerordentlich entmutigend.

Wir wollen der Methode volle Gerechtigkeit widerfahren lassen und nur, was unzweideutig bewiesen ist, anerkennen, respektive ablehnen; danach müssen wir die Edebohlsschen und unsere Ergebnisse zu folgenden drei Schlubfolgerungen zusammenfassen:

1. Die Dekapsulation der Nieren ist bei schwerem Morbus Brightii eine gefährliche Operation (Beweis: $26 \%$ operative Mortalität).

2. Dauerheilungen sind in keinem Fallevon sicherem Morbus Brightii erzielt worden.

3. Es sind Besserungen erzielt worden:

Objektiv ist in einem Falle das Fortbleiben der sonst leicht auftretenden Oedeme zu verzeichnen, dagegen keine fortschreitende Besserung der Albuminurie und Zylindurie.

Subjektiv in einem andern Falle das Fortbleiben von Kopfschmerzen und Flimmern vor den Augen, welches vor der Operation bestanden hatte.

Meinem hochverehrten Chef, Herrn Prof. Israel, gestatte ich mir auch an dieser Stelle für die Anregung zu dieser Arbeit und seine wertvolle Unterstïtzung bei ihrer Anfertigung meinen ergebensten Dank auszusprechen. 